\title{
Making holograms in middle and high schools
}

\section{Tung Jeong}

Tung $\mathrm{H}$. Jeong, "Making holograms in middle and high schools," Proc. SPIE 3831, Sixth International Conference on Education and Training in Optics and Photonics, (16 June 2000); doi: 10.1117/12.388736

SPIE Event: Education and Training in Optics and Photonics (ETOP'99), 1999, Cancun, Mexico 


\title{
Making Holograms In Middle and High Schools
}

\author{
Tung $\mathbf{H}$. Jeong \\ Lake Forest College \\ Center for Photonics Studies \\ 555 North Sheridan Road \\ Lake Forest, IL 60045 USA \\ jeong@LFC.edu
}

\begin{abstract}
Holography is a worthy topic that should become an integral part of any basic science curriculum. It embodies basic scientific principle that include the direct applications of three Nobel Prize physics concepts; it involves procedures that teaches the scientific method of problem solving; it can be learned by "doing" without previous experience; it is artistically creative; it can be appreciated by students of all ranges of abilities; and it is an open-ended subject so that specially interested students can continue to pursue deeper and more creative projects beyond the scope that fits into the curriculum. Finally, with the availability of high quality and low cost diode lasers, it is an affordable unit for any school.
\end{abstract}

Keywords: Holography, optics education, pedagogy, diode laser holography

\section{INTRODUCTION}

Human kind had evolve from the Stone Age to the Bronze Age, and is now in transition from the Electric Age to the Age of Light. Just as the transistor ushered in the electronic revolution, the laser is bringing us to the new era of photonics.

Holography is the most natural subject to introduce to our young students the concepts of photonics because its study includes all the basic principles of light. The central concepts of three Nobel Prizes in physics are directly related to holography: Lippmann (1908), Bragg (1915), and Gabor (1971). Yet, it can be studied with enthusiasm using low cost diode lasers and relatively safe chemicals.

Herein we discuss details of projects in making and understanding holography. The cost of equipment is no longer a factor. Through a graphical model ${ }^{(1)}$, many basic principles of interference and diffraction can be taught without advanced mathematics.

\section{A DIODE LASER SYSTEM FOR MAKING HOLOGRAMS}

Recently, some class IIIa "laser pointers" are available in popular stores at prices as low as $\$ 7.99^{(2)}$. whose output has surprisingly long coherence length after a warmup period of about 10 minutes. By removing from such a laser the collimation lens, the output is spread out in a highly eccentric elliptical profile. This renders the laser much safer for use by young students because the power that can enter a human eye is greatly reduced, to a level below that when one looks at the scanner operating in a grocery store.

Such a wide spread of light not only make it safer, it also can be used to make holograms with minimum additional optical elements. Furthermore, the output has no interference or diffraction patterns associated with light that have traveled through multiple surfaces with dirt.

We found by random sampling that laser pointers that has the shape of a bullet generally has a feedback circuit that stabilizes the frequency, giving the output a long coherence length. Pen-shaped lasers do not the same circuit, and their outputs are unstable.

The lens must be removed very carefully. Heating can destroy the laser; and care must be taken as not to introduce any dust into the laser. We have done it by slowly sawing away the lens manually. 
Because the batteries that accompany the laser pointers are small and have short useable life, we generally remove the battery housing as well, and connect the laser to two D-batteries in series. By using 2 D-batteries which provides 3.0 volts (instead of the maximum intended 4.5 volts), a stable 3 milliwatts power output is obtained. This will provide many hours of stable operation with extended laser life.

There is an alternative to dissection a laser pointer. A class IIIa diode laser is available along with the same control circuit, with a removable collimating lens and two wires for external connections to batteries ${ }^{(3)}$.

\section{PROJECTS IN HOLOGRAPHY}

Our goals here are:

1. To show how to use the "laser pointer" without any modification to make a Denisyuk hologram viewable with a point source of incandescent light. In this case, a concave mirror is needed to spread out the light, which often costs more than the laser;

2. To remove the collimating lens and make a superior hologram of the same type, without the need of a "beam-spreader." Furthermore, the battery housing can also be removed and substituted by two D batteries;

3. To use the naturally diverging beam with a highly eccentric elliptical profile to make panoramic transmission and reflections holograms viewable with the same laser light.

4. To make high dispersion diffraction gratings.

\subsection{Using a "laser pointer" to make reflection holograms}

Figure 1 shows a configuration for making a white light viewable reflection hologram using the laser pointer along with

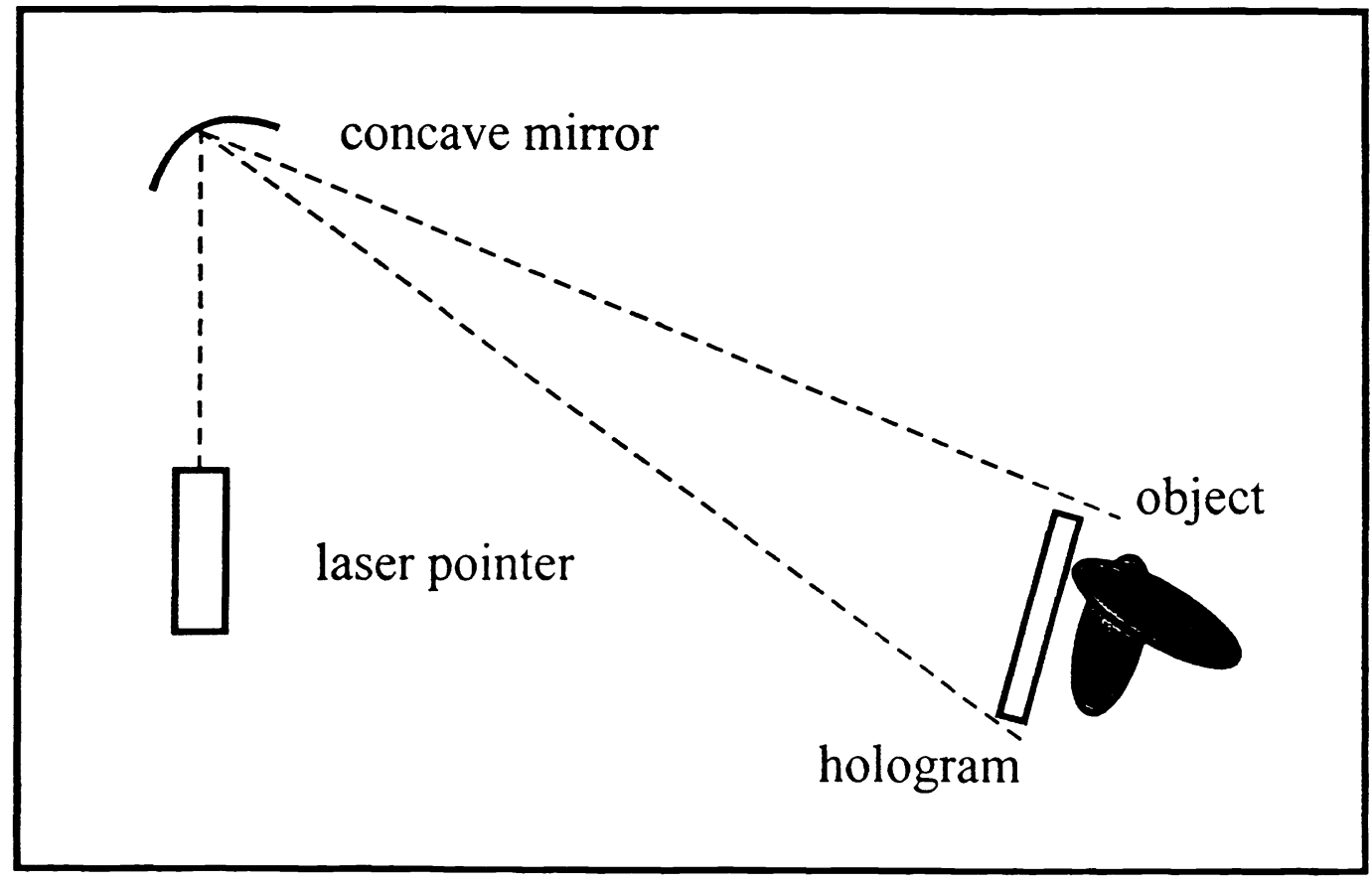

Figure 1

Using a laser pointer "as is" requires a concave mirror to focus and then spread out the beam for making holograms. 
minimum equipment. Use two common clothespins to support the laser; one to hold down the "on" switch and the other to form the third leg so that the system is its own tripod. Direct the beam at a concave front surfaced mirror (3) ("beamspreader").

Finally, a solid object (not much larger than the $6.35 \mathrm{~cm} \times 6.35 \mathrm{~cm}$ holographic plates to be used) is illuminated by the spreading beam.

The entire system is set on a solid surface such as a kitchen counter, a laboratory table, the floor, or a calm sandy beach (at night). With enough "ordinary light" allowed to provide minimal visibility after dark adaptation, the spreading laser beam is directed at the object. Adjust the distances and orientations of all elements until the object is illuminated as evenly as possible. Now block the beam near the laser with a "shutter" (a cardboard, for example) to prevent the light from reaching the object. A holographic plate (Slavich PFG-01M) ${ }^{(3)}$ is placed in contact with the object, with the emulsion (sticky) side facing it. After a settling time of a few seconds, an exposure of $\mathbf{5}$ seconds is made by lifting and lowering the "shutter". If there is air movement or too much ambient light, a box (with the inside painted black) should be placed over the entire system.

The plate is developed using a chemical kit, called JD-3, ${ }^{(3)}$ according to instructions that accompanies it. The steps include: develop in a tray until opaque (1-2 minutes), rinse briefly in distilled water, bleach (copper sulphate) until completely clear, rinse again until all yellow color is gone, develop again in vitamin C with light on (sunlight is best, but any bright light will do) until the hologram turns brown, rinse and then allow the hologram to dry. Squeegee or hair blower may be used to shorten drying time. The thoroughly dried hologram can then be viewed using a pen flashlight or any point incandescent source. This particular chemical process minimizes emulsion shrinkage and allows the red image to be viewed with the spreading laser light as well, with increased image resolution.

However, because the collimating lens has two surfaces, their reflections form interference patterns on the spread out beam. Also, dirt collected on the lens and concave mirror causes diffraction patterns. Thus, the above hologram is expected to have a mottled pattern across its surface.

\subsection{Using a diode laser without collimator for making reflection holograms}

By removing the built-in collimating lens from the "pointer," the remaining unit emits light that spreads out in an elliptical cone. Here the beam is completely "clean" as if it had been spatially filtered, and the exposure over the holographic plate is more even. We no longer need the concave mirror. Holograms made with this laser are free of mottled patterns.

Furthermore, one can remove the small and expensive batteries, and connect the bare laser unit to two 1.5 volts $\mathrm{D}$ batteries in series (important: the housing is + and the electrical lead inside is -). The laser is normally operated at 4.5 volts. By applying only 3.0 volts, the laser still yields over $2.5 \mathrm{~mW}$ and its life expectancy is greatly extended. Such a minimal laser is commercially available ${ }^{(3)}$.

To make a white light reflection hologram with absolutely no optical equipment except for the laser itself: support the laser at the previous location of the concave mirror and rotate it until the elliptical beam has its long axis in the vertical position; record the same type of hologram with the exposure time of about 5 seconds. The hologram is much superior to the ones made in Figure 1 because the direct laser light that served as reference beam has no interference or diffraction patterns.

\subsection{Using the elliptical beam to make panoramic transmission holograms}

We can take advantage of its highly eccentric elliptical beam and make transmission holograms with extraordinary wide angles of view.

Figure 2 shows the configuration used for making a deep scene panoramic transmission hologram. A sandbox (or a calm beach at night) is constructed using white silica sand (available in hardware stores) supported by eight "lazy balls."(3) These balls have zero coefficient of restitution at $23^{\circ} \mathrm{C}$ and provide a rigid base that both minimizes vibration and wobbling. The laser is placed at one corner with its beam spreading across the sand.

In a darkroom with minimal indirect green light, cut the holographic film into rectangular pieces (approximately $4 \times 10 \mathrm{~cm}$ )using a paper cutter (or scissor). Sandwich the horizontal strip of film between two larger glass plates and hold the left and right sides with strong steel paper clamps. 


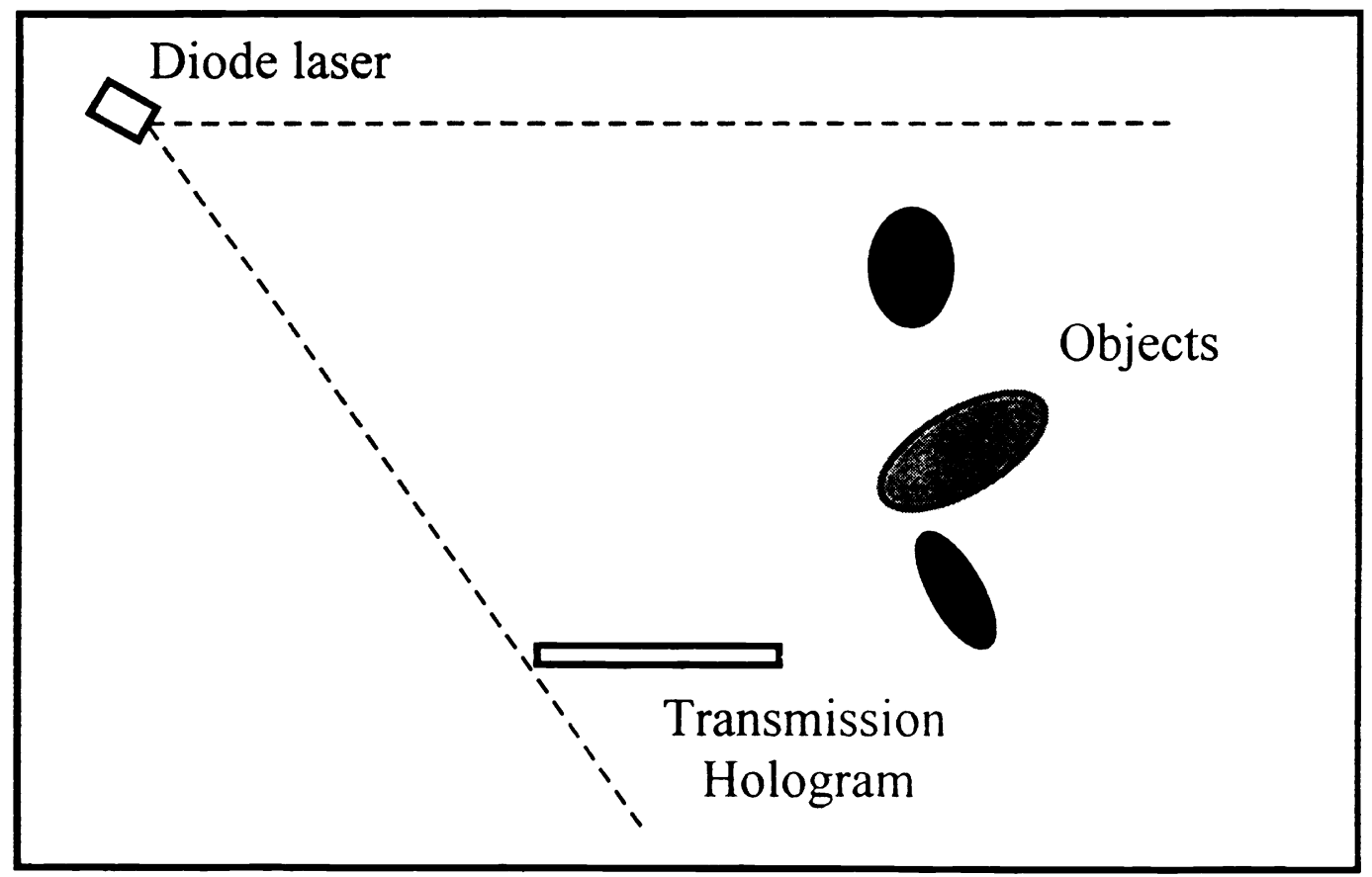

Figure 2

Making a transmission hologram using a diode laser requires absolutely no optical equipment. The highly eccentric elliptical beam serves as reference beam on one side, and as object beam on the other side.

Place several objects across one side of the spread out beam and stick a white cardboard (the size of the film sandwich) at the future location of the film. Note that the polarization of the laser light is horizonta7l. The film (cardboard) should be positioned so that the incident angle is about $57^{\circ}$, near the Brewster's's angle, thus avoiding the "thin film interference" effects caused by the internal reflections.

Arrange all the components so that both the objects and the cardboard are well illuminated by the spreading beam. Now block the laser light by sticking another cardboard near its output end. Remove the cardboard that represent the film and sticking the film sandwich into the sand at that location. Waiting for at least five minutes for the trapped air in the sandwich to escape before exposure. It is advisable to turn off all the light (except the laser, whose light is blocked) during the waiting period unless the system can be covered.

The exposure is about 30 seconds. The hologram is developed the same way as before, and the virtual image is viewed by light from the same laser under the same configuration as during the exposure. The real image of the hologram can be projected onto a white screen if a collimated laser beam is directed at the hologram in a backward direction. The screen should be located at the approximate position of the objects.

The above setup configuration has one drawback: all the objects are side-lit. To make holograms with objects illuminated more from the front, a front surface mirror larger then the hologram is needed.

Figure 3 shows the arrangement. The mirrored is oriented to direct a reference beam to the recording film which faces the objects being frontally illuminated. A black cardboard is used as a baffle to prevent light from reaching the back of the film.

\subsection{Making diffraction gratings with high dispersion}

Figure 4 shows the configuration in which a hologram is made by recording the interference pattern of two beams that meet at $90^{\circ}$ on the film. The pattern is essentially that of Young's double slit interference, with the slits separated so far that the spacing of 


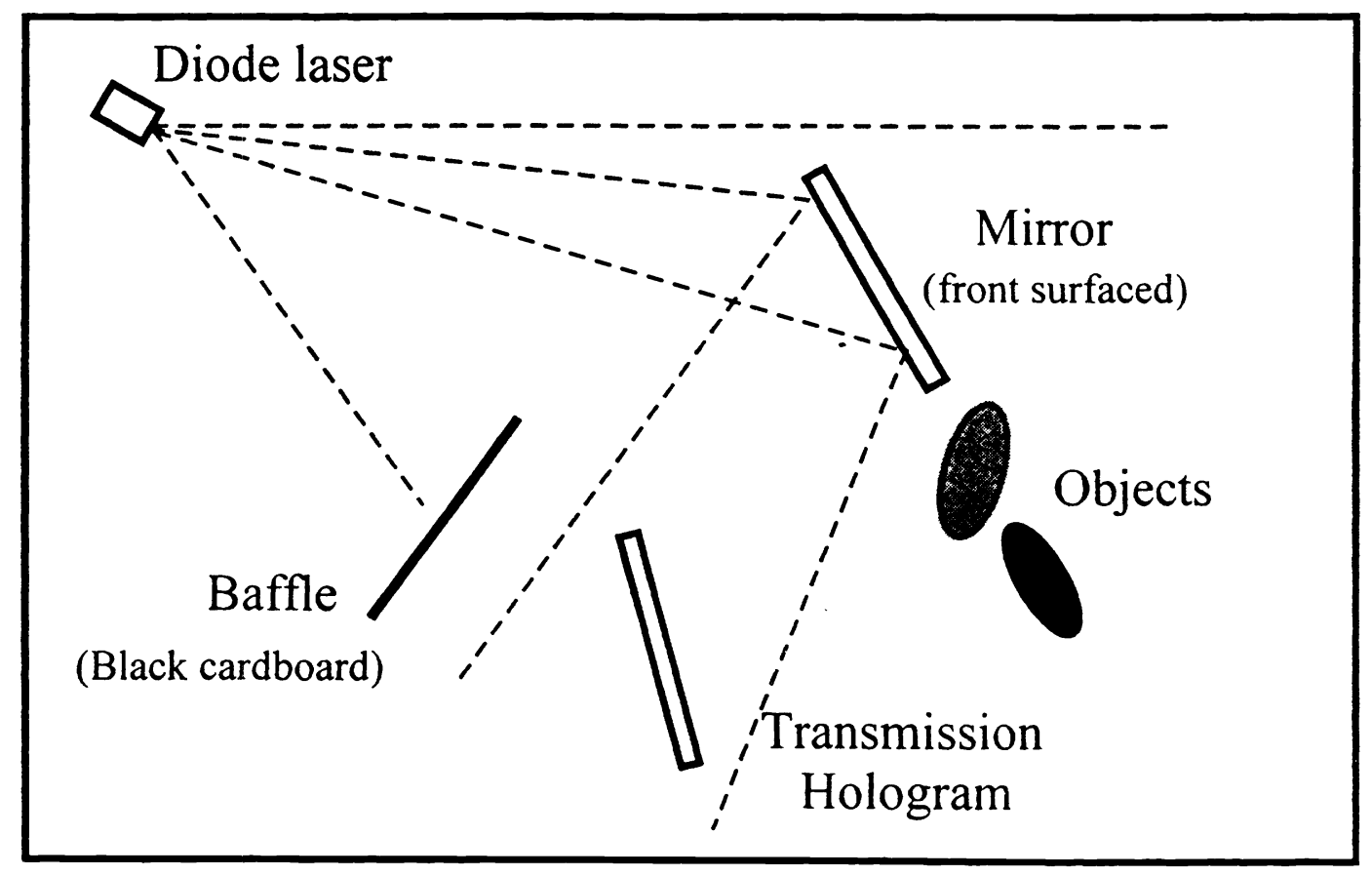

Figure 3

By using a front surfaced mirror, the reference beam can be redirected so that the objects can be illuminated from the front.

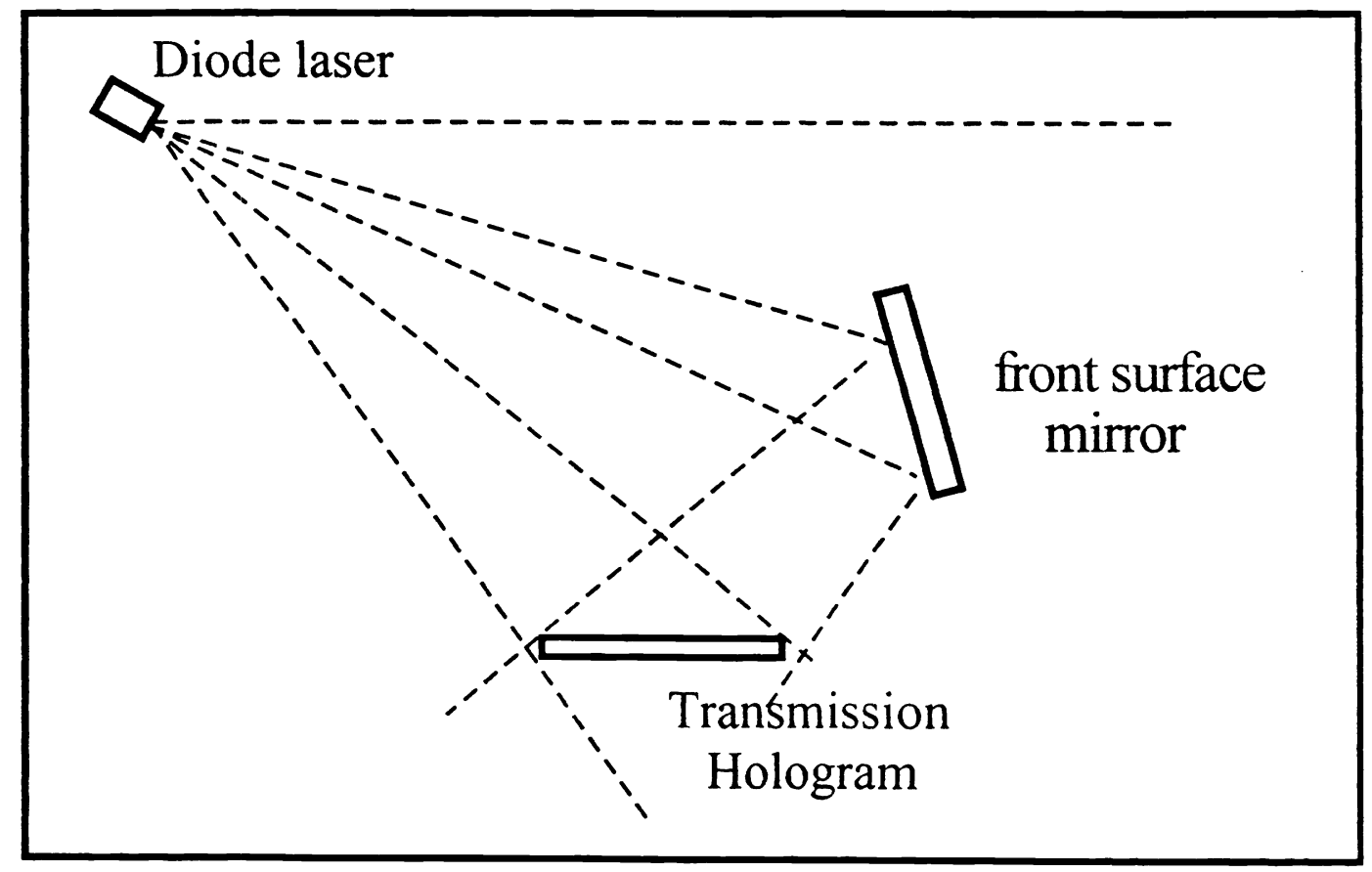

Figure 4

To make a highly dispersive holographic diffraction grating, the object beam is redirected by a mirror to interfere with the reference beam at $90^{\circ}$. 
the fringes is equal to the wavelength.

When sunlight is passed through a narrow slit formed by two razor blades and is incident onto the grating at $45^{\circ}$, a widely spread out spectrum can be observed along with the Fraunhofer absorption line. The grating is also extremely useful for observing the spectrum of any low pressure discharges.

\section{Conclusions}

Over the past two decades, many schools have adopted holography into the science curriculum. What prevented the expansion of this program is cost and safety. Helium-Neon laser costs several bundred dollars; and collimated beams are often mischievously used by student to point at people. With the availability of low cost diode lasers with the collimating lens removed, both obstacles are now overcome.

\section{References}

1. Tung H. Jeong, “A Geometric Model for Holography”, Am. J. Phys., August, 714-717 (1975)

2. Eckard Drug Stores has sold such a laser pointer for \$7.99. Office Depot sells one for \$9.99.

3. Integraf is a source for class IIIa diode lasers with a removable collimator and electric leads for external power source and a supplier of holographic plates, film, developing chemicals, and "lazy balls". E-mail: integraf@aol.com; Fax: 847-615-0835; Website: $\mathbf{h t t p}: / /$ members.aol.com/integraf. 